\title{
PERFIL DE IDOSOS SUBMETIDOS À AVALIAÇÃO GERIÁTRICA AMPLA EM SERVIÇO DE REABILITAÇÃO
}

\author{
Profile of older people submitted to comprehensive geriatric assessment in a rehabilitation \\ service
}

\section{Perfil de ancianos sometidos a una amplia evaluación geriátrica de un servicio de rehabilitación}

Fernanda Silva Rocha

Pontifícia Universidade Católica de Goiás - PUC - Goiânia (GO) - Brasil

Giulliano Gardenghi

Pontifícia Universidade Católica de Goiás - PUC - Goiânia (GO) - Brasil

Patrícia Conceição Oliveira

Universidade Federal de Goiás - Goiânia (GO) - Brasil

\section{RESUMO}

Objetivo: Descrever o perfil dos idosos submetidos à avaliação geriátrica ampla em um serviço de reabilitação. Métodos: Estudo documental, retrospectivo, do tipo descritivo, realizado em um serviço de reabilitação de Goiânia, Goiás, Brasil, com pacientes idosos que foram submetidos à avaliação geriátrica ampla no período de dezembro de 2014 a dezembro de 2015. Informações sobre sexo, idade, estado civil, arranjo familiar, escolaridade, índice de massa corporal, polifarmácia, incontinência urinária e atividade física foram coletadas através de dados de prontuário e analisadas estatisticamente. Resultados: A média de idade da população foi de 70,4 anos $( \pm 7,8)$ onde $67,45 \%$ ( $\mathrm{n}=228$ ) dos avaliados são do sexo feminino, 50,29\% $(\mathrm{n}=170)$ apresenta escolaridade de $1-4$ anos, 44,38\% (n=150) são casados, 56,21\% (n=190) moram com familiares e 77,51\% ( $\mathrm{n}=262)$ são sedentários. A incontinência urinária no sexo feminino é prevalente em 49,53\% ( $\mathrm{n}=107)$, ainda que seja relevante no sexo masculino $21,81 \%(\mathrm{n}=24)$. Fazem uso de polifarmácia $48,82 \%(\mathrm{n}=165)$ dos avaliados, $77,51 \%(\mathrm{n}=262)$ são sedentários e 59,17\% (n=171) estão acima do peso. Conclusão: O perfil é de idosos jovens, do sexo feminino, sedentários, com baixa escolaridade, casados, que moram com familiares e apresentam sobrepeso. A maior prevalência de incontinência urinária e polifarmácia foi encontrada entre pessoas do sexo feminino.

Descritores: Avaliação Geriátrica; Serviços de Saúde para Idosos; Perfil de Saúde.

\section{ABSTRACT}

Objective: To describe the profile of older people submitted to comprehensive geriatric assessment in a rehabilitation service. Methods: Retrospective documentary descriptive study conducted at a rehabilitation service in Goiânia, Goiás, Brazil, with older patients submitted to Comprehensive Geriatric Assessment from December 2014 to December 2015. Information on gender, age, marital status, family arrangement, education, body mass index, polypharmacy, urinary incontinence, and physical activity were collected from medical records and submitted to statistical analysis. Results: The mean age of the population was 70.4 years $( \pm 7.8)$. In all, $67.45 \%$ ( $n=228$ ) of the participants are women, $50.29 \%(n=170)$ have $1-4$ years of study, $44.38 \%(n=150)$ are married, $56.21 \%(n=190)$ live with relatives, and $77.51 \%(n=262)$ are sedentary. Urinary incontinence is prevalent in $49.53 \%(n=107)$ of the women, although it is quite relevant in men, with a rate of $21.81 \%(n=24)$. In all, $48.82 \%(n=165)$ of the participants presented polypharmacy, $77.51 \%(n=262)$ are sedentary and $59.17 \%(n=171)$ are overweight. Conclusion: Most participants are young old, women, sedentary, have low education, are married, live with relatives, and are overweight. The highest prevalence of urinary incontinence and polypharmacy occurs in women.

Descriptors: Geriatric Assessment; Health Services for the Aged; Health Profile. 


\section{RESUMEN}

Objetivo: Describir el perfil de los ancianos sometidos a una amplia evaluación geriátrica de un servicio de rehabilitación. Métodos: Estudio documental, retrospectivo, del tipo descriptivo realizado en un servicio de rehabilitación de Goiânia, Goiás, Brasil, con pacientes mayores que fueron sometidos a una amplia evaluación geriátrica en el período entre diciembre de 2014 y diciembre de 2015. Las informaciones sobre el sexo, la edad, el estado civil, el orden familiar, la escolaridad, el indice de masa corporal, la polifarmacia, la incontinencia urinaria y la actividad fisica fueron recogidas a través de datos de los historiales clínicos y analizadas estadísticamente. Resultados: La media de edad de la población fue de 70,4 años $( \pm 7,8)$ en el cual el 67,45\% $(n=228)$ de los evaluados son del sexo femenino, el 50,29\% ( $n=170)$ presenta escolaridad entre 1-4 años, el 44,38\% ( $n=150)$ son casados, el 56,21\% $(n=190)$ viven con sus familiares y el $77,51 \%(n=262)$ son sedentarios. La incontinencia urinaria del sexo femenino es prevalente en el 49,53\% ( $n=107)$ y el 21,81\% ( $n=24)$ en el sexo masculino. El $48,82 \%(n=165)$ de los evaluados usan la polifarmacia, el 77,51\% ( $n=262)$ son sedentarios y el 59,17\% ( $n=171)$ tienen el peso elevado. Conclusión: El perfil es de ancianos jóvenes, del sexo femenino, sedentarias, con baja escolaridad, casadas, que viven con sus familiares y tienen sobrepeso. La mayor prevalencia de incontinencia urinaria y polifarmacia ha sido encontrada entre las personas del sexo femenino.

Descriptores: Evaluación Geriátrica; Servicios de Salud para Ancianos; Perfil de Salud.

\section{INTRODUÇÃO}

Devido à transição sociodemográfica, o Brasil vem passando por um processo natural de envelhecimento populacional ${ }^{(1)}$. Acredita-se que, em 2025, o país será o sexto do mundo em número absoluto de idosos, totalizando 33,8 milhões de indivíduos nessa faixa etária, sendo o crescimento do segmento de idosos velhos, aqueles com mais de 80 anos, o mais expressivo ${ }^{(2)}$.

O envelhecimento populacional aliado à má alimentação, ao sedentarismo, à obesidade e ao consumo de álcool e de tabaco trouxe uma maior prevalência de doenças crônicas, com consequente aumento no número de complicações clínicas que causam perda funcional e diminuição na qualidade de vida, fato que modifica a demanda por políticas públicas tornando necessária a elaboração de indicadores de saúde capazes de reconhecer os agravos que impactam a saúde dos idosos ${ }^{(3)}$.

É típico nas pessoas de idade avançada o sobrepeso e a obesidade, a incontinência urinária, a polifarmácia e o sedentarismo que culminam com perda da função e da qualidade de vida do idoso ${ }^{(4)}$. A incontinência urinária, por exemplo, é definida como a condição na qual a perda involuntária de urina é um problema social ou higiênico e é objetivamente demonstrada. É prevalente entre os idosos e impacta na funcionalidade, com efeitos deletérios à saúde, além de reflexos negativos no sistema público de saúde ${ }^{(5,6)}$.

O uso de medicamentos constitui-se na atualidade uma epidemia entre idosos, seja em decorrência do aumento exponencial das doenças crônicas e de suas sequelas, seja pelo poderio e marketing da indústria farmacêutica, além de uma formação profissional na área da saúde com muita ênfase na medicalização(7). Muito se estuda em relação às consequências do uso de medicações nessa população e sabe-se que existe um maior risco de eventos adversos e associação com maior morbimortalidade ${ }^{(8)}$. A polifarmácia, definida como consumo de cinco ou mais medicamentos concomitantes, é um desafio para a geriatria devido ao risco de eventos adversos aumentarem em 13\% com o uso de dois agentes e de $58 \%$ quando este número aumenta para cinco, elevando-se para $82 \%$ nos casos em que são consumidos sete ou mais medicamentos ${ }^{(9)}$.

Estudos epidemiológicos sobre o estado nutricional em idosos indicam que o sobrepeso e a obesidade estão associados com o risco de morbidade e de mortalidade, mas pouco se sabe sobre a relação entre índice de massa corporal (IMC) e envelhecimento. O IMC ainda é considerado um bom indicador do estado nutricional em estudos epidemiológicos, apesar de não haver consenso quanto ao ponto de corte do IMC mais adequado para idosos, já que eles apresentam modificações da composição corporal quando comparados à idade adulta. A Organização Mundial da Saúde (OMS) salienta a necessidade de indicar os valores de referência de peso corporal no envelhecimento, entre diferentes idades e gêneros, em razão da tendência em aumentar o IMC até a meia-idade, estabilizar-se ao atingir a faixa de 65 anos para homens e 75 anos para mulheres, e redução após essa fase ${ }^{(10)}$.

A relação entre a atividade física e a saúde do idoso tem sido muito estudada e já é consenso o papel fundamental na manutenção da capacidade funcional, sendo decisório o tipo correto de atividade prescrito. O sedentarismo está associado à condição de saúde, maior incidência de doenças crônicas e pior funcionalidade ${ }^{(11)}$.

A multiplicidade das afeç̧ões que acometem os idosos inclui aspectos clínicos, psicológicos e sociais que interagem entre si, modificando a exteriorização das doenças e exigindo uma abordagem diferenciada. O envelhecimento frequentemente acarreta alterações funcionais insidiosas, e os instrumentos de rastreamento de condições desencadeadoras de declínio funcional permitem intervenções precoces de maior impacto potencial e possibilidade de melhor planejamento das ações na área da saúde ${ }^{(2)}$.

A avaliação geriátrica ampla (AGA) é um termo utilizado para descrever o exame das diversas funções do paciente idoso. Identificar as doenças crônicas, o nível de independência e a autonomia, os recursos financeiros disponíveis para aquisição de 
serviços e a existência, ou não, de suporte familiar e social fazem parte dessa avaliação. A AGA não é uma avaliação isolada, e sempre deve resultar em uma intervenção, seja ela de reabilitação, de aconselhamento, seja de indicação de internação em hospital, ou instituição de longa permanência. Essa avaliação difere do exame clínico padrão por enfatizar a avaliação da capacidade funcional e da qualidade de vida, além de se basear em escalas e testes quantitativos e padronizados ${ }^{(2)}$.

Estudos epidemiológicos adquirem importância nesse cenário por possibilitarem a identificação dos determinantes e dos fatores etiológicos do envelhecimento, propiciando um melhor entendimento das diferentes situações clínicas e uma melhor assistência à saúde dessas populações, buscando um prolongamento da vida humana por meio da prevenção das doenças e da promoção à saúde ${ }^{(4)}$.

Um estudo epidemiológico é essencial para o conhecimento da população trabalhada, sendo ferramenta importante na gestão dos serviços de saúde e da atuação profissional. Epidemiologia é o estudo da frequência, da distribuição e dos determinantes dos estados ou eventos relacionados à saúde ${ }^{(12)}$. Este conhecimento traz subsídios para a capacitação das pessoas e comunidades para modificarem os determinantes sociais, através de ações específicas, visando benefícios para a qualidade de vida ${ }^{(4)}$.

Diante disso, objetivou-se descrever o perfil dos idosos submetidos à avaliação geriátrica ampla em um serviço de reabilitação.

\section{MÉTODOS}

Trata-se de um estudo documental, retrospectivo, do tipo descritivo, realizado por meio de coleta de dados dos prontuários físicos dos pacientes idosos submetidos à avaliação geriátrica ampla (AGA) em um serviço de reabilitação na cidade de Goiânia, Goiás, Brasil. Realizou-se o levantamento dos dados epidemiológicos de todos os pacientes abordados pela AGA no período de dezembro de 2014 a dezembro de 2015. O número de prontuários avaliados foi de 393 , excluindo-se 55 incompletos, totalizando 338 prontuários.

Realizou-se a pesquisa no Ambulatório do Hospital de Dermatologia Sanitária e Reabilitação Santa Marta (HDS), localizado na Rodovia GO 403, na cidade de Goiânia. Tal unidade presta assistência ambulatorial em geriatria exclusivamente para pacientes do Sistema Único de Saúde (SUS), através do controle da Central de Regulação da Secretaria Municipal de Saúde de Goiânia.

A AGA é realizada na instituição por equipe multiprofissional composta por médico geriatra, fisioterapeuta, enfermeiro, terapeuta ocupacional, assistente social, fonoaudiólogo, psicólogo, nutricionista, farmacêutico e cirurgião-dentista. São utilizados instrumentos padronizados pelo hospital, baseados nas recomendações do Ministério da Saúde ${ }^{(13)}$, e os resultados são anotados nas fichas resumo dos prontuários, as quais, anotadas no período mencionado, constituem a base para a coleta de dados da presente pesquisa.

O levantamento nos arquivos da instituição gerou a listagem dos pacientes que foram abordados pela AGA no período analisado. A planilha de coleta de dados é composta por variáveis sociodemográficas (sexo, idade, estado civil, arranjo familiar e escolaridade), marcadores nutricionais (peso, altura e índice de massa corporal) e outras condições de saúde (existência de continência urinária, polifarmácia e prática de exercício físico). A amostra constitui-se de idosos não institucionalizados que buscaram atendimento ambulatorial.

Agrupou-se a variável arranjo familiar em: mora com família, mora com outras pessoas (amigos ou cuidadores) e mora sozinho. A escolaridade é estabelecida de acordo com a quantidade de anos que o idoso declarou ter frequentado a escola (0 anos ou analfabeto, de 1 a 4 anos, 5 a 8 anos ou acima de 8 anos), devido serem as faixas padronizadas para avaliação do estado cognitivo do idoso ${ }^{(14)}$.

O estado nutricional foi definido pelo IMC, calculado através da mensuração da altura e do peso corporal do idoso: peso (quilos)/altura (metros) ao quadrado. Os idosos foram classificados em baixo peso (IMC $<18,5 \mathrm{~kg} / \mathrm{m}^{2}$ ), eutróficos (IMC $22,0-$ $25,0 \mathrm{~kg} / \mathrm{m}^{2}$ ), excesso de peso (IMC $>25 \mathrm{~kg} / \mathrm{m}^{2}$ ) e obesidade $\left(\text { IMC }>30 \mathrm{~kg} / \mathrm{m}^{2}\right)^{(10)}$.

Para a classificação da continência urinária, questionou-se ao idoso sobre a ocorrência de perdas involuntárias de urina. De acordo com a resposta, fez-se a categorização em continentes (sem perdas involuntárias de urina) ou incontinentes (com perdas involuntárias de urina) ${ }^{(15)}$.

De acordo com o inventário do uso diário de medicamentos feito pelo idoso avaliado, o farmacêutico o classificou com presença de polifarmácia (consumo de 5 ou mais medicamentos concomitantes) ou ausência de polifarmácia (consumo de menos que 5 medicamentos concomitantes) ${ }^{(9)}$.

Considerou-se a mensuração da prática de exercício físico de acordo com o relato do idoso. Considerou-se exercício físico a prática regular (mínimo três vezes por semana, com duração mínima de 30 minutos) ou estruturada, tais como caminhadas, dança, atividades de grupos de terceira idade, hidroginástica/natação, musculação e outros exercícios em academias e práticas esportivas $^{(16)}$.

Manipularam-se os dados de forma padronizada por meio de análise estatística descritiva, sendo os dados apresentados em porcentagem, em média e em desvio-padrão. As variáveis categóricas estão expressas em números absolutos e relativos. Aplicou-se o qui-quadrado de tendência linear. 
Realizou-se a pesquisa em conformidade com a Resolução 466/12 do Conselho Nacional de Saúde, que regulamenta as normas de pesquisa com seres humanos, tendo aprovação do Comitê de Ética em Pesquisa Humana e Animal da Pontifícia Universidade Católica de Goiás (PUC GO) com aprovação no Parecer número 1.509.986.

\section{RESULTADOS}

O número e os percentuais sociodemográficos (idade, estado civil, escolaridade, relacionamento familiar) dos idosos submetidos à avaliação geriátrica ampla (AGA) do serviço de reabilitação avaliados na presente pesquisa estão dispostos na Tabela I.

Tabela I - Descrição das características sociodemográficas dos idosos submetidos à avaliação geriátrica ampla. Goiânia, Goiás, Brasil, 2015.

\begin{tabular}{llcc}
\hline Variável & & $\mathbf{n}$ & $\mathbf{\%}$ \\
\hline Sexo & Feminino & 228 & 67,46 \\
Faixa etária & Masculino & 110 & 32,54 \\
& Abaixo de 60 anos & 2 & 0,58 \\
& $60-69$ anos & 175 & 51,78 \\
& $70-79$ anos & 111 & 32,84 \\
& $80-89$ anos & 44 & 13,02 \\
Estado civil & Acima de 90 anos & 6 & 1,78 \\
& & & \\
& Casados & 150 & 44,38 \\
& Viúvos & 105 & 31,06 \\
Escolaridade & Divorciados ou separados & 41 & 12,13 \\
& Solteiros & 28 & 8,28 \\
& Não informado & 14 & 4,15 \\
& Analfabeto & & 17,75 \\
& $1-4$ anos & 60 & 50,30 \\
& $5-8$ anos & 170 & 14,49 \\
Arranjo familiar & Acima de 8 anos & 49 & 11,83 \\
& Não informado & 40 & 5,62 \\
& Mora com família & 19 & 56,21 \\
& Mora com outras pessoas & 190 & 21,9 \\
& Mora sozinho & 74 & 6,38 \\
\hline & Não informado & 53 & 21 \\
\hline
\end{tabular}

A média de idade da população investigada é de 70,4 anos $( \pm 7,8)$, sendo que o mais jovem tem 57 anos e o mais velho tem 96 anos. Destaca-se que dois pacientes da amostra apresentavam idade inferior a 60 anos de idade, mas se submeteram à AGA devido ao quadro de fragilidade apresentado. Observou-se ainda a predominância de pacientes do sexo feminino, sendo 67,46\% do sexo feminino ( $\mathrm{n}=228)$ e $32,54 \%$ do sexo masculino $(\mathrm{n}=110)$.

Quanto ao estado civil, 44,38\% dos idosos ( $\mathrm{n}=150)$ declararam-se casados, sendo 31,06\% $(\mathrm{n}=105)$ viúvos, 12,13\% ( $\mathrm{n}=41)$ separados ou divorciados, $8,28 \%(n=28)$ solteiros e $4,15 \%(n=14)$ não informaram o seu estado civil.

Observou-se baixa escolaridade entre idosos entrevistados, sendo que $68,05 \%(n=230)$ são analfabetos, ou apresentam escolaridade de até quatro anos de estudos, $11,83 \%(\mathrm{n}=40)$ dos idosos informaram escolaridade acima de 8 anos.

Em relação às questões de relacionamento familiar e de criação de novas redes sociais, observou-se que $56,1 \%(n=190)$ dos idosos moram com familiares, $21,9 \%(\mathrm{n}=74)$ mora com amigos, cuidadores e outros. O número de idosos que residem sozinhos é relevante e chama a atenção, quando $15,38 \%(\mathrm{n}=53)$ declararam essa situação.

Quanto à prática de exercício físico, observou-se, através do autorrelato registrado em prontuário, que 77,51\% ( $\mathrm{n}=262)$ dos idosos da amostra declararam não fazer exercício físico, $13,31 \%(\mathrm{n}=45)$ dos idosos na faixa etária dos 60-69 anos praticavam atividade física. Os praticantes acima de 70 anos de idade equivalem a 7,99\% ( $\mathrm{n}=27)$, conforme demonstrado na Tabela II. 
Tabela II - Descrição da realização de exercício em idosos submetidos à avaliação geriátrica ampla. Goiânia, Goiás, Brasil, 2015.

\begin{tabular}{llcc}
\hline \multicolumn{1}{c}{ Variável } & & $\mathbf{n}$ & $\mathbf{\%}$ \\
\hline Amostra & Não sedentário & 72 & 21,30 \\
& Sedentário & 262 & 77,51 \\
Exercício físico e saúde & Não informado & 4 & 1,18 \\
& & & 13,91 \\
Exercício físico e faixa etária & Feminino & 47 & 7,40 \\
& Masculino & 25 & 0 \\
& & 0 & 13,31 \\
& Abaixo de 60 anos & 45 & 5,62 \\
& $60-69$ anos & 19 & 2,07 \\
& $70-79$ anos & 7 & 0,30 \\
\hline
\end{tabular}

Quando questionados sobre os episódios de perda involuntária de urina, 38,76\% ( $\mathrm{n}=131)$ declararam apresentá-los, sendo assim considerados incontinentes. Observou-se uma predominância da incontinência urinária (IU) no sexo feminino, com 46,93\% (n=107) das idosas. No sexo masculino, a incontinência urinária esteve presente em $21,81 \%(\mathrm{n}=24)$. A maior prevalência da incontinência urinária proporcional à faixa etária mostra-se entre 70 a 79 anos, com $47,75 \%(n=53)$ dos idosos desta faixa de idade se declarando incontinentes. A descrição dos dados referentes à continência urinária está apresentada na Tabela III.

Tabela III - Distribuição da incontinência urinária em idosos submetidos à avaliação geriátrica ampla. Goiânia, Goiás, Brasil, 2015.

\begin{tabular}{llcc}
\hline \multicolumn{1}{c}{ Variável } & & n & \% \\
\hline Amostra & Continentes & 206 & 60,94 \\
& Incontinentes & 131 & 38,76 \\
Incontinência por sexo & Não informado & 1 & 0,29 \\
& & & \\
Incontinência por faixa etária & Feminino & 107 & 46,93 \\
& Masculino & 24 & 21,81 \\
& & & \\
& Abaixo de 60 anos & 0 & 0 \\
& $60-69$ anos & 55 & 31,42 \\
& $70-79$ anos & 53 & 47,75 \\
& $80-89$ anos & 20 & 45,45 \\
& Acima de 90 anos & 3 & 50 \\
\hline
\end{tabular}

A prática da polifarmácia é frequente na população estudada. Observou-se, conforme Tabela IV, que 48,82\% ( $\mathrm{n}=165$ ) dos idosos avaliados apresentavam polifarmácia, sendo mais frequente entre as idosas, com um percentual de $52,19 \%$ ( $\mathrm{n}=119)$. Realizou-se a análise estatística através do teste qui-quadrado, onde o valor P é de 0,097 , não evidenciando correlação estatisticamente significativa entre os sexos. Quanto à faixa etária, a polifarmácia esteve mais presente no grupo etário de 60-69 anos de idade. 
Tabela IV - Distribuição da polifarmácia em idosos submetidos à avaliação geriátrica ampla. Goiânia, Goiás, Brasil, 2015.

\begin{tabular}{llll}
\hline Polifarmácia & & n & \% \\
\hline Prática de polifarmácia & & 165 & 48,82 \\
& Com polifarmácia & 47,04 \\
& Sem polifarmácia & 4,14 \\
Polifarmácia por sexo & Não informado & 14 & 52,19 \\
& & & 41,82 \\
Polifarmácia por faixa etária & Feminino & 119 & 0,61 \\
& Masculino & 46 & 44,24 \\
& $>60$ anos & 1 & 38,18 \\
& $60-69$ anos & 73 & 15,15 \\
\end{tabular}

$\mathrm{Na}$ presente pesquisa avaliou-se ainda o índice de massa corporal (IMC) de 289 idosos por meio do peso dividido pela altura ao quadrado. Observou-se, por meio deste, que 37,71\% (n=109) dos idosos são eutróficos. O excesso de peso e a obesidade estiveram presentes em 59,17\% (n=171) idosos. A prevalência de baixo peso deste estudo foi de 3,12\% (n=9), sem diferença significativa entre os sexos. O exposto está apresentado na Tabela V.

Tabela V - Descrição do índice de massa corporal (IMC) em idosos submetidos à avaliação geriátrica ampla (AGA). Goiânia, Goiás, Brasil, 2015.

\begin{tabular}{|c|c|c|c|c|}
\hline \multicolumn{2}{|c|}{ Variável } & \multicolumn{2}{|c|}{$\mathbf{n}$} & $\%$ \\
\hline \multicolumn{5}{|l|}{ Amostra } \\
\hline & Magreza & \multicolumn{2}{|c|}{9} & 3,12 \\
\hline & Eutrófico & \multicolumn{2}{|c|}{109} & 37,71 \\
\hline & Excesso de peso & \multicolumn{2}{|c|}{105} & 36,33 \\
\hline & Obesidade & \multicolumn{2}{|c|}{66} & 22,84 \\
\hline IMC & Magreza & Eutrófico & Excesso de peso & Obesidade \\
\hline \multicolumn{5}{|l|}{$>60$ anos } \\
\hline $60-69$ anos & $5(1,72 \%)$ & $45(15,52 \%)$ & $56(19 ., 1 \%)$ & $46(15,86 \%)$ \\
\hline 70-79 anos & $3(1,03)$ & $42(14,48 \%)$ & $34(11,72 \%)$ & $17(5,86 \%)$ \\
\hline $80-89$ anos & $1(0,34 \%)$ & $20(6,90 \%)$ & $13(4,48 \%)$ & $1(0,34 \%)$ \\
\hline$>90$ anos & $0(0 \%)$ & $2(0,69 \%)$ & $1(0,34 \%)$ & $1(0,34 \%)$ \\
\hline \multirow[t]{5}{*}{ Sexo } & & \multicolumn{2}{|c|}{ Masculino } & Feminino \\
\hline & Magreza & \multicolumn{2}{|c|}{$4(1,18 \%)$} & $5(1,48 \%)$ \\
\hline & Eutrófico & \multicolumn{2}{|c|}{$41(12,13 \%)$} & $68(20,11 \%)$ \\
\hline & Excesso de peso & \multicolumn{2}{|c|}{$35(10,35 \%)$} & $70(20,71 \%)$ \\
\hline & Obesidade & \multicolumn{2}{|c|}{$12(3,55 \%)$} & $54(15,98 \%)$ \\
\hline
\end{tabular}

\section{DISCUSSÃO}

No que tange às características sociodemográficas da presente pesquisa, houve predominância de pacientes do sexo feminino, o que também foi evidenciado em outros estudos devido ao processo denominado feminização do envelhecimento populacional, com maior probabilidade de sobrevida para as mulheres ${ }^{(17,18)}$. No entanto, apesar de sua sobrevida ser maior quando comparada aos homens, as mulheres estão mais susceptíveis às incapacidades e aos múltiplos problemas de saúde nas idades mais longevas. Tal condição exige atenção especial na elaboração de políticas públicas que atendam às demandas específicas dessa população, proporcionando assim o envelhecimento com qualidade de vida, bem como a prevenção de problemas de saúde $^{(1)}$. Em relação à predominância de idosos na faixa etária entre 60 a 69 anos de idade, a amostra da presente pesquisa reflete a realidade da proporção de idosos por faixa etária segundo censo do Instituto Brasileiro de Geografia e Estatística ${ }^{(19,20)}$.

A baixa escolaridade é outra característica marcante na presente pesquisa, encontrando-se que há idosos possuidores de um período de até quatro anos de escolaridade, fato convergente com a informação levantada pelo censo quanto à escolaridade dos idosos responsáveis pelos domicílios no Brasil ${ }^{(20)}$. Tal constatação leva à reflexão quanto à necessidade de implementação de 
ações para melhoria da mesma, já que o analfabetismo pode levar à maior dependência do idoso de outras pessoas e à piora da sua autonomia e qualidade de vida, além da maior inatividade física ${ }^{(21)}$.

Segundo dados do Censo Populacional, 10,9\% das pessoas idosas viviam sozinhas, e, através da Pesquisa Nacional por Amostra de Domicílios (PNAD) realizada no ano de 2014, constatou-se que essa proporção havia subido, sendo estimada em quase $14,4 \%^{(22)}$. Tal percentual está próximo ao encontrado na presente pesquisa, que totaliza $15,38 \%$. Esse fato não significa necessariamente um problema, já que pode ser uma opção do idoso, entretanto essa condição representa estado de risco tendo em vista a possibilidade de perda da autonomia, risco de solidão e vulnerabilidade ${ }^{(23)}$. Um ponto importante seria a investigação social da população avaliada com vistas a analisar os impactos dessa situação na saúde dos idosos ${ }^{(24)}$.

O nível de exercício físico apresentado na presente pesquisa é um dado preocupante, já que é um indicador importante de saúde da população. A inatividade física é responsável direta por 6\% dos casos de doenças coronarianas, 7\% de diabetes tipo II, $10 \%$ de câncer de mama e por 5,3 milhões de mortes ocorridas, além de sua associação com a menor capacidade de mobilidade corporal e maior fragilidade, especialmente em indivíduos idosos ${ }^{(25)}$. Um estudo longitudinal das consequências para a saúde dos diferentes níveis de aptidão física, realizado com em 25.341 homens e 7.080 mulheres, revelou que uma baixa aptidão aeróbica era um precursor mais importante de mortalidade do que as doenças acima referidas ${ }^{(26)}$.

A recomendação de 150 minutos de atividade física moderada na semana ${ }^{(27)}$ é acatada por $18 \%$ dos homens e $14 \%$ das mulheres de 65 a 74 anos. No presente estudo, 13,31\% dos idosos na faixa etária dos 60-69 anos praticavam atividade física. Os praticantes acima de 75 anos são $8 \%$ dos homens e $4 \%$ das mulheres ${ }^{(28)}$, o que vai ao encontro da amostra do presente estudo, sendo que 4,44\% das mulheres acima de 75 anos praticavam atividade física apesar da discordância quanto ao sexo masculino (3,25\% relataram praticá-la). É oportuno colocar que a literatura aponta que a prática do exercício físico está mais presente entre os idosos do sexo masculino ${ }^{(27)}$.

As novas diretrizes da atividade física para a promoção da saúde recomendam que os indivíduos realizem atividade física de intensidade moderada por, pelo menos, 30 minutos por dia, na maior parte dos dias da semana (de preferência todos), de forma contínua ou acumulada ${ }^{(28)}$.

O envelhecimento predispõe à incontinência urinária (IU), o que pode levar a um quadro clínico de depressão, de isolamento e de envergonhamento, alterando o convívio social e e impactando na mobilidade do idoso ${ }^{(15,29)}$. Não há consenso na literatura quanto às taxas de prevalência de IU encontradas nos diversos estudos entre os idosos, as quais variam de $8,5-55 \%{ }^{(15)}$. A divergência entre os números pode estar relacionada aos fatores culturais, às características sociodemográficas, aos hábitos de vida ou mesmo aos instrumentos utilizados nas pesquisas ${ }^{(29)}$.

Sabe-se que a prevalência de IU eleva-se proporcionalmente ao lado da idade, sendo o principal fator de risco nos homens para alterações patológicas da próstata ${ }^{(30)}$. Quando se considera a idade, a literatura cita a maior prevalência entre os 70-75 $\operatorname{anos}^{(29)}$, dados que se aproximam dos resultados encontrados na presente amostra, exceto pela ausência de relação direta do aumento dos episódios de incontinência com o avançar da idade. Em relação ao sexo, a literatura aponta que a IU é três vezes maior em mulheres quando comparadas aos homens por conta de questões anatômicas, hormonais e de paridade, sendo que a frequência de incontinentes entre os homens varia de $10-15 \%{ }^{(15)}$. Na presente pesquisa, o sexo feminino é responsável pelo maior número de incontinentes.

O uso diário de cinco ou mais medicamentos pelo idoso, técnica denominada polifarmácia, é uma prática corriqueira. Estudos no Brasil apontam uma prevalência de 5-27\%, sendo a sua utilização mais comum entre as idosas e em idade mais avançada $^{(9,31)}$. Fato semelhante também ocorre em outro estudo, discordando apenas da faixa etária, pois a maior prevalência de polifarmácia encontrada ocorreu no grupo etário de 60-69 anos de idade. As conclusões divergentes sugerem que a polifarmácia tem características regionais ${ }^{(31,32)}$.

O perfil farmaco-epidemiológico da presente pesquisa indica a necessidade de desenvolver práticas de cuidados de saúde para garantir a segurança do paciente, sendo ainda importante considerar o baixo nível educacional dos idosos brasileiros uma vez que este é um dos fatores que interferem na adesão a um tratamento farmacológico, no uso de medicamentos impróprios e na polifarmácia ${ }^{(33)}$

A prevalência de baixo peso do presente estudo é de 3,1\%, sem diferença significativa entre os sexos. O dado é inferior aos apresentados na teoria, que chegam a $6,7 \%$, sendo $8,9 \%$ para homens e $4,9 \%$ mulheres $^{(23)}$.

Observa-se, no atual estudo, a predominância de idosos jovens (60-69 anos) com excesso de peso e obesidade, com declínio progressivo do peso nas faixas etárias consecutivas, o que converge com outros estudos na área ${ }^{(34)}$. Essa diferença pode ser caracterizada pela perda de motoneurônios e menor densidade óssea, evidente nos mais idosos, sem desconsiderar o fator de relação entre a ingestão calórica e o gasto energético. É de suma importância o conhecimento do perfil descrito para que medidas de ação direcionadas possam ser planejadas e executadas visando à prevenção e promoção da saúde dos idosos da comunidade ${ }^{(34)}$.

É oportuno salientar algumas limitações da presente pesquisa, como o fato de ser uma pesquisa baseada em prontuários dos pacientes, não sendo possível a observação clínica para possíveis correlações, bem como o acesso aos dados das comorbidades da população. Outra limitação se refere ao fato de não ter sido avaliado o tipo de exercício físico e sua regularidade para um melhor conhecimento do nível de aptidão física dos idosos avaliados. 


\section{CONCLUSÃO}

O perfil dos idosos submetidos à avaliação geriátrica ampla em um serviço de reabilitação compreende idosos jovens (60-69 anos) do sexo feminino com baixa escolaridade, casadas, fazendo o uso de polifarmácia e acima do peso. Em se tratando de questões de relacionamento familiar e de criação de novas redes sociais, constatou-se a predominância da residência com familiares e sedentários. A maior prevalência de incontinência urinária e polifarmácia foi encontrada nos idosos do sexo feminino, mas ainda é relevante no sexo masculino.

\section{REFERÊNCIAS}

1. Küchemann BA. Envelhecimento populacional, cuidado e cidadania: velhos dilemas e novos desafios. Soc Estado. 2012;27(1):165-80.

2. Lino VTS, Portela MC, Camacho LAB, Rodrigues NCP, Andrade MKN, O'Dwyer G. Rastreamento de problemas de idosos na atenção primária e proposta de roteiro de triagem com uma abordagem multidimensional. Cad Saúde Pública. 2016;32(7):1-12.

3. Duncan BB, Chor D, Aquino EML, Bensenor IM, Mill JG, Schmidt MI, et al. Doenças Crônicas Não Transmissíveis no Brasil: Prioridade para enfrentament e investigação. Rev Saúde Pública. 2012;46(Supl 1):126-34.

4. Confortin SC, Jayce I, Schneider C, Antes DL, Cembranel F, Ono LM, et al. Condições de vida e saúde de idosos : resultados do estudo de coorte EpiFloripa Idoso. Epidemiol Serv Saúde. 2017;26(2):499-510.

5. Marques LP, Schneider IJC, Giehl MWC, Antes DL, D'Orsi E. Demographic, health conditions and lifestyle factors associated with urinary incontinence in elderly from Florianopolis, Santa Catarina, Brazil. Rev Bras Epidemiol. 2015;18(3):595-606.

6. Barentsen JA, Visser E, Hofstetter H, Maris AM, Dekker JH, de Bock GH. Severity, not type, is the main predictor of decreased quality of life in elderly women with urinary incontinence: a population-based study as part of a randomized controlled trial in primary care. Health Qual Life Outcomes. 2012;10(1):153.

7. Ramos LR, Tavares NUL, Bertoldi AD, Farias MR, Oliveira MA, Luiza VL, et al. Polypharmacy and Polymorbidity in Older Adults in Brazil: a public health challenge. Rev Saúde Pública. 2016;50(Supl 2):1-13.

8. Baldoni AO, Ayres LR, Martinez EZ, Dewulf NLS, Santos V, Obreli-Neto PR, et al. Pharmacoepidemiological profile and polypharmacy indicators in elderly outpatients. Brazilian J Pharm Sci. 2013;49(3):443-52.

9. Silva R, Schmidt O, Silva S. Polifarmácia em geriatria. Rev AMRIGS. 2012;56(2):164-74.

10. Souza R, Fraga JS, Gottschall CBA, Busnello FM, Rabito EI. Avaliação antropométrica em idosos: estimativas de peso e altura e concordância entre classificações de IMC. Rev Bras Geriatr Gerontol. 2013;16(1):81-90.

11. Gillespie LD, Robertson MC, Gillespi WJ. Interventions for preventing falls in older people living in the community. Cochrane. 2012;(9).

12. Lima-Costa MF, Barreto SM. Tipos de estudos epidemiológicos: conceitos básicos e aplicacações na área do envelhecimento. Epidemiol Serv Saúde. 2003;12(4):189-201.

13. Ministério da Saúde (BR). Cadernos e atenção básica envelhecimento e saúde da pessoa idosa. Brasília: Ministério da Saúde; 2006.

14. Brucki SMD, Nitrini R, Caramelli P, Bertolucci PHF, Okamoto IH. Sugestões para o uso do mini-exame do estado mental no Brasil. Arq Neuropsiquiatr. 2003;61(3B):777-81.

15. Honório MO, Santos SMA. Incontinência urinária e envelhecimento: impacto no cotidiano e na qualidade de vida. Rev Bras Enferm. 2009;62(1):51-6.

16. Tribess S, Virtuoso JS Jr. Prescrição de exercícios físicos para idosos. Rev Saúde Com. 2005;1(2):163-72.

17. Closs VE, Ziegelmann PK, Gomes I, Schwanke CHA. Frailty and geriatric syndromes in elderly assisted in primary health care. Acta Sci Heal Sci. 2016;38(1):9-25.

18. Gray WK, Richardson J, McGuire J, Dewhurst F, Elder V, Weeks J, et al. Frailty Screening in Low- and Middle-Income Countries: A Systematic Review. J Am Geriatr Soc. 2016;64(4):806-23.

19. Lima-Costa MF, Matos DL, Camargos VP, Macinko J. Tendências em dez anos das condições de saúde de idosos brasileiros: evidências da Pesquisa Nacional por Amostra de Domicílios (1998, 2003, 2008). Ciênc Saúde Coletiva. 2011;16(9):368996. 
20. Instituto Brasileiro de Geografia e Estatística - IBGE. Perfil dos estados e municípios brasileiros. Brasília: IBGE; 2014.

21. Pilger C, Menon MU, Mathias TAF. Utilização de serviços de saúde por idosos vivendo na comunidade. Rev Esc Enferm USP. 2013;47(1):213-20.

22. Instituto Brasileiro de Geografia e Estatística - IBGE. Síntese de indicadores sociais: uma análise das condições de vida da população brasileira: estudos e pesquisas. Rio de Janeiro: IBGE; 2015.

23. Wosiack R, Berlim CS, Santos GA. Fatores de risco e de proteção evidenciados em idosos de Ivoti-RS : intervenções psicossociais na área da Gerontologia. Rev Bras Ciênc Envelhecimento Hum. 2013;10(3):256-70.

24. Perseguino MG, Horta ALM, Ribeiro CA. A família frente a realidade do idoso de morar sozinho. Rev Bras Enferm. 2017;70(2):251-7.

25. Santos VR, Gomes IC, Santos LL, Agostinete RR, Freitas IF Júnior. Associação entre fatores de risco cardiovascular e capacidade funcional de idosos longevos. Med (Ribeirão Preto). 2013;46(1):10-6.

26. Trevizani GA, Benchimol-Barbosa PR, Nadal J. Efeitos da idade e da aptidão aeróbica na recuperação da frequência cardíaca em homens adultos. Arq Bras Cardiol. 2012;99(3):802-10.

27. Moreira RM, Teixeira RM, Novaes KO. Contribuições da atividade física na promoção da saúde, autonomia e independência de idosos. Rev Kairós. 2014;17(1):201-17.

28. Matsudo SM, Matsudo VKR, Barros TL Neto. Atividade física e envelhecimento: aspectos epidemiológicos. Rev Bras Med Esporte. 2001;7(1):2-13.

29. Marques LP, Schneider IJC, Giehl MWC, Antes DL, D’Orsi E. Demographic, health conditions, and lifestyle factors associated with urinary incontinence in elderly from Florianopolis, Santa Catarina, Brazil. Rev Bras Epidemiol. 2015;18(3):595-606.

30. Marques SR. Tratamento fisioterapêutico na incontinência urinária em idosas. Rev Saúde Integrada. 2016;17(9):110-6.

31. Duarte LR, Gianinni RJ, Ferreira LR, Aparecida M, Galhardo SD. Habits of medication consumption among elderly SUS and health insurance users. Cad Saúde Coletiva. 2012;20(1):64-71.

32. Ribas C, Oliveira KR. Perfil dos medicamentos prescritos para idosos em uma Unidade Básica de Saúde do município de Ijuí-RS. Rev Bras Geriatr Gerontol. 2014;17(1):99-114.

33. Silveira EA, Dalastra L, Pagotto V. Polypharmacy, chronic diseases and nutritional markers in community-dwelling older. Rev Bras Epidemiol. 2014;17(4):818-29.

34. Paludetti L, Traldi Z. A influência da massa corporal em idosos caidores e idosos não caidores. Rev Kairós Gerontol. 2014;17(4):157-73.

\section{Endereço do primeiro autor:}

Fernanda Silva Rocha

Pontifícia Universidade Católica de Goiás/ PUC/CEAFI Pós Graduação

Rua T-28, 1806

Bairro: Setor Bueno

CEP 74215-040 - Goiânia - GO - Brasil

E-mail: fernandarocha_13@hotmail.com

\section{Endereço para correspondência:}

Giulliano Gardenghi

Pontifícia Universidade Católica de Goiás/Centro de Estudos Avançados e Formação Integrada - PUC/CEAFI - Pós

Graduação

Rua T-28, 1806

Bairro: Setor Bueno

CEP 74215-040 - Goiânia - GO - Brasil

E-mail: coordenacao.cientifica@ceafi.com.br 